\title{
Healing Mechanism and Osteogenic Capacity of Bovine Bone Mineral-Human Amniotic Mesenchymal Stem Celland Autogenous Bone Graft in Critical Size Mandibular Defect
}

\author{
David B. Kamadjaja ${ }^{1,2}$, Purwati2,3,4, Fedik A. Rantam ${ }^{2,3}$, Ferdiansyah ${ }^{3,5}$, D. Coen Pramono ${ }^{1}$ \\ ${ }^{1}$ Department of Oral \& Maxillofacial Surgery, Faculty of Dentistry, Airlangga University, Surabaya, Indonesia \\ 'Laboratory of Stem Cell, Institute of Tropical Disease, Airlangga University, Surabaya, Indonesia \\ ${ }^{3}$ Regenerative Medicine \& Stem Cell Centre, Airlangga University \& Dr. Soetomo General Hospital, Surabaya, \\ Indonesia \\ ${ }^{4}$ Department of Internal Medicine, Dr. Soetomo General Hospital, Surabaya, Indonesia \\ ${ }^{5}$ Department of Orthopaedic \& Traumatology, Dr. Soetomo General Hospital, Surabaya, Indonesia \\ Email: davidbk65@gmail.com
}

Received 21 September 2015; accepted 24 October 2015; published 27 October 2015

Copyright $@ 2015$ by authors and Scientific Research Publishing Inc.

This work is licensed under the Creative Commons Attribution International License (CC BY). http://creativecommons.org/licenses/by/4.0/

(c) (i) Open Access

\section{Abstract}

Experiments on maxillofacial bone tissue engineering showed the promising result; however, its healing mechanisms and effectiveness had not been fully understood. The aim of this study is to compare the bone healing mechanism and osteogenic capacity between bovine bone mineral loaded with hAMSC and autogenous bone graft in the reconstruction of critical size mandibular bone defect. Critical size defects were made at the mandible of $45 \mathrm{New}$ Zealand white rabbits reconstructed with BBM-hAMSC, BBM alone, and ABG, respectively. At the end of first, second, and twelfth weeks, five rabbits from each experimental week were sacrificed for histology and immunohistochemistry staining. Expressions of vascular endothelial growth factor (VEGF), bone morphogenic proteins-2 (BMP2), Runx2 and the amount of angiogenesis were analyzed in the first and second week groups, while expressions of Runx2, osteocalcin, collagen type-I fibres, trabecular area and bone incorporation were analyzed in the twelfth week groups. The result showed that expressions of VEGF, BMP2 and Runx2 as well as the amount of angiogenesis were higher in ABG compared with BBM-hAMSC group in the first and second weeks of healing. The result of twelfth week of healing showed that expressions of Runx2 and osteocalcin as well as the thickness of collagen type-I fibres were significantly higher in BBM-hAMSC compared to ABG group, while there was no statistically difference in trabecular area and bone incorporation between BBMhAMSC and ABG group. This study concluded that early healing activities were higher in auto- 
genous bone graft than in BBM-hAMSC, while osteogenic activities in the late stage of healing were higher in BBM-hAMSC compared to autogenous bone graft. It was also concluded that the osteogenic capacity of BBM-hAMSC was comparable to autogenous bone graft in the reconstruction of critical size defect in the mandible.

\title{
Keywords
}

\author{
Bone Healing Mechanism, Osteogenic Capacity, Human Amniotic Mesenchymal Stem Cell, Bovine \\ Bone Mineral, Autogenous Bone Graft, Critical Size Mandibular Bone Defect
}

\section{Introduction}

Surgical reconstruction of critical size mandibular defect has been a great challenge in oral and maxillofacial surgery. Gold standard for mandibular reconstruction is autogenous bone graft. However, autogenous bone graft has limitation in shape, size, and its availability; furthermore, it has been attributed to donor site morbidity [1] [2]. Bone tissue engineering using mesenchymal stem cell (MSC) is a potential alternative to autogenous bone graft. Experimental studies proved that tissue engineering used to reconstruct segmental defects in long bones [3]-[7] and mandibles [8] [9] showed promising result. However, the healing mechanisms of composite MSCscaffold and their osteogenic capacity in the reconstruction of critical size mandibular defects, have not been fully understood until today. The study of healing mechanism of mandibular defects reconstructed with composite MSC-scaffold can be performed by analyzing the amount of angiogenic and osteogenic differentiation processes observed in the scaffolds, while the osteogenic capacity of this approach may be evaluated by the amount of new bone trabeculae formed in the scaffold and the incorporation between newly formed bone and the recipient bone.

Mesenchymal stem cell-based bone tissue engineering generally uses autologous bone marrow-derived mesenchymal stem cell (BM-MSC) due to its highest osteogenic capacity among other type of MSC [10]. However, it has limitations as it requires invasive procedure to collect bone marrow aspirates which is a potential morbidity to the patients; the quality of MSC largely depends on the patient general health condition and age. Allogeneic MSC, therefore, is now being studied as an alternative to autologous BM-MSC. Human amniotic membrane mesenchymal stem cell (hAMSC), as one of the sources of allogeneic MSC, has recently been explored because of its pluripotential [11] [12] and low immunogenic properties [13] [14]. Application of hAMSC in bone tissue engineering, however, has not been reported in the literature.

The aim of this study is to compare the early and late phase of healing mechanism and osteogenic capacity between composite bovine bone mineral scaffold loaded with hAMSC and autogenous bone graft, the gold standard of bone graft material, in the reconstruction of critical size mandibular defect.

\section{Material and Methods}

\subsection{In Vitro Procedure}

\subsubsection{Isolation of Human Amniotic Membrane}

The procurement of human amniotic membrane was harvested from Cesarean section performed at Dr. Soetomo General Hospital, Surabaya which was legally and ethically approved by Committee for Ethics on Health Researches, Dr. Soetomo General Hospital, Surabaya. The procedure for isolation and culture of hAMSC were performed at Laboratory of Stem Cell, Institute of Tropical Disease, Airlangga University, Surabaya using modified Alviano’s protocol as described in our previous paper [15].

\subsubsection{Cell Culture}

The cells collected were cultured on dishes using Dulbecco's DMEM/F12 (1:1) (Gibco BRL, Gaithersburg, MD, USA), supplemented with human leukemia inhibitory factor (10 ng/mL) and fetal bovine serum (Gibco BRL). The cell adherence to the dish was evident at day 3 and on the seventh day when the culture reached $80 \%$ confluence (Figure 1), cell splittings were done, half to two thirds of the cells were subsequently replated using the same medium. 


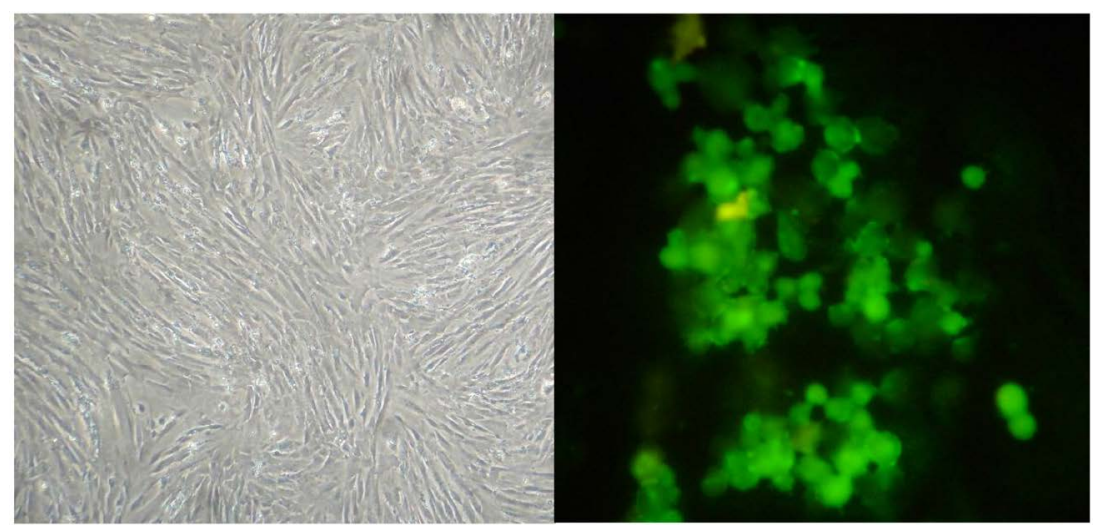

(a)

(b)

Figure 1. Culture and phenotypic characterization of human amniotic MSC. Upon 7 days of culture, fibroblast-like cell cells were adherent to the plate reaching $80 \%$ confluence (a) (inverted microscope, 200× magnification). The immunofluorescence result showed that the cells strongly expressed CD 105 (stained in green), one of the MSC specific markers (b).

\subsubsection{Phenotypic Characterization}

The MSC phenotypic characterization was performed with immunostaining as follows. Cultured cells were plated onto coverslips, incubated at $37^{\circ} \mathrm{C}$ for 1 - 2 hours, fixed with formaldehyde $10 \%$ for 15 minutes, and then rinsed four times with PBS. The cells were blocked with PBS and FBS 1\% for 15 - 30 minutes. FITC-labelled mouse monoclonal antibody anti-human CD 105 and CD 45 was applied to the culture and incubated for 60 minutes. Thereafter, the cells were rinsed with PBS twice and analyzed using fluoresence microscope. The positive MSC marker, CD 105, was showed by green staining expressed by the cells (Figure 1) as opposed to dark, negative haematopoeitic marker when stained with anti-CD 45.

\subsection{In Vivo Procedure}

Forty five New Zealand white rabbit were divided into 3 groups based on the time of observation, which were first, second, and twelfth week. Each group was subsequently divided into three experimental groups which were BBM-hAMSC, BBM alone (BBM), and autogenous bone graft (ABG), consisting of 5 rabbits per group. Critical size defects were surgically created in the rabbits' mandibles which were reconstructed with BBMhAMSC, BBM alone, and ABG, respectively. Fifteen rabbits from each experimental weekwere sacrificed for histology and immunohistochemistry staining. Expression of vascular endothelial growth factor (VEGF), bone morphogenic proteins-2 (BMP2), Runx2 and amount of angiogenesis were analyzed in the first and second week groups, while expression of Runx2, osteocalcin, collagen type-I fibres, trabecular area and bone incorporation were analyzed in the twelfth week groups.

\subsubsection{Surgical Procedure}

The surgical procedure was done at operating room at Laboratory of Stem Cell, Institute of Tropical Disease, Airlangga University which had been approved by Animal Care and Use Committee (ACUC) Faculty of Veterinary Medicine, Airlangga University. Forty-five male, New Zealand white rabbit, aged 6 months, weighing $3.0-3.5 \mathrm{~kg}$, were used in this study. They were divided into 3 groups based on the time of observation which are one, two, and twelve weeks, in each of the group they are further subdivided into 3 experimental groups based on methods of defect reconstruction i.e. scaffold bovine bone mineral and hAMSC (BBM-hAMSC), scaffold BBM alone (BBM), and autogenous bone graft (ABG), making a total of 9 groups consisting of 5 rabbits per group.

Rabbits were anaesthetized with intramuscular injection of ketamine $\mathrm{HCl}$ at a dose of $20 \mathrm{mg}$ per $\mathrm{kg}$ body weight. A critical size defect measuring $5 \times 10 \times 4 \mathrm{~mm}$ was made at the inferior border of right side of the mandible using straight-end drill under cupious saline irigation. The defects in the first experimental group were reconstructed with BBM-hAMSC, while in the second and third group the defects were filled with BBM alone 
and autogenous bone graft harvested from iliac crestal bone of the same rabbit, respectively. Fixation of the implanted material was achieved by suturing the overlying muscle under slight tension. Soft tissue and skin suturing was done with absorbable suturing material.

\subsubsection{Specimen Preparation}

Fifteen rabbits were sacrificed after first, second and twelve weeks to collect the specimens for histology and immunohistochemistry staining. The specimens collection was done by removing the scaffold or bone graft including the surrounding host bone of 2 - $3 \mathrm{~mm}$ wide using sharp straight-end drill. They were fixed in $10 \%$ buffered formaldehyde solution for 3 days and then decalcified with $10 \%$ ethylenediaminetetraacetic acid until 30 days before embedding in paraffin. Sections, $5 \mu \mathrm{m}$ thick, were deparaffinized with xylene, rehydrated in $100 \%$ alcohol, and washed in distilled water and then stained with hematoxylin-eosin.

\subsubsection{Immunohistochemical Staining}

Sections for immunohistochemical staining were incubated in 3\% peroxide for 30 minutes acid to block endogenous peroxidase, soaked in 0.025\% trypsin-phosphate buffer saline for 6 minutes and finally washed with 3 times for 2 minutes. Sections were stained with anti VEGF-A antibody (anti-rabbit VEGF polyclonal antibody, USCN, USA; followed by anti-human VEGF monoclonal antibody, LifeSpan BioSciences, Inc. USA), anti BMP2 antibody (anti-rabbit BMP-2 polyclonal antibody, ABIN, USA; followed by anti-human BMP-2 monoclonal antibody, LifeSpan BioSciences, Inc. USA), anti-rabbit Runx-2 monoclonal antibody (Santa Cruz Biotech., USA), anti-rabbit osteocalcin monoclonal antibody (Novus Biological, USA), and anti-rabbit collagen I monoclonal antibody (Novus Biological, USA).

\subsubsection{Data and Statistical Analysis}

The data of VEGF, BMP-2, Runx2 and Osteocalcin expressions were counted as the number of cells positively stained with the respective antibodies, while Collagen type-I thickness was represented by the thickness (in $\mu \mathrm{m}$ ) of bone matrix positively stained with collagen type-I antibody. The histology sections were used for evaluation of the number of vascular tissues (angiogenesis), the area of bone trabeculae, and bone incorporation between the newly formed bone and the resipient bone. The evaluation of bone incorporation used modified LaneShandu scoring system ( 0 = non-union, 1 = fibrous union, 2 = fibro-osseous union, $3=$ osseous union, $4=$ mature and complete ossoeus union).

Statistical analysis was performed using software package SPSS version 16 (IBM corp). Data from each experiment group were statistically analyzed with the assumption of homogeneity of variances and normal distribution of errors being tested for the variables examined. One-way analysis of variance followed by multiple comparison test (Tukey HSD) and Kruskal Wallis followed by Mann-Whitney test were used to show the significance among the groups $(p<0.05)$.

\section{Result}

\subsection{Microscopic Healing of Critical Size Mandibular Bone Defects}

The results of evaluation at the first and second week post implantation were as follows. The microscopy of histology staining of the first week evaluation showed obvious amorphous structures in the centre of the scaffold in both BBM-hAMSC and BBM group as opposed to mature trabecular bone in ABG group. However, it was evident that at this stage more advanced tissue healing was found in BBM-hAMSC and ABG group compared to delayed healing in BBM group, characterized by lack of mesenchymal tissue and the cells populating the scaffolds being predominantly of polymorphonuclear cells, mononuclear cells and erythrocytes indicating prolonged tissue inflammation. Histology findings at second week of implantation showed comparable tissue healing in BBM-hAMSC and BBM group, however osteoid matrices were found in the former group. The ABG group showed mesenchymal and osteoid tissue blended with mature trabecular bone indicating activities of bone absorption and formation. Findings at twelfth week showed that islands of bony trabeculaes being in maturation processes were predominant in BBM-hAMSC group which were blended with mesechymal and osteoid tissues compared to predominant mesenchymal and osteoid tissue with scarce, streaky bony trabeculae in BBM group. The ABG group showed relatively mature bony trabeculaes incorporating with more mature surrounding bone, areas of mesenchymal and osteoid tissue were also seen in between those trabecular bones (Figure 2). 


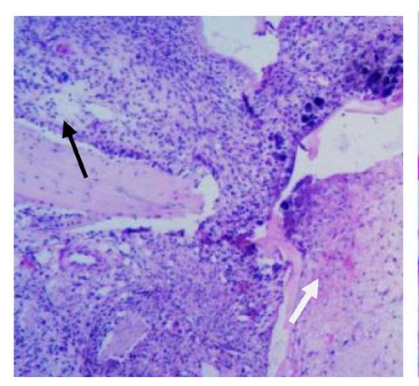

(a)

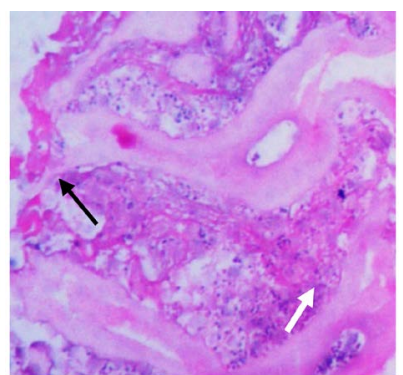

(b)

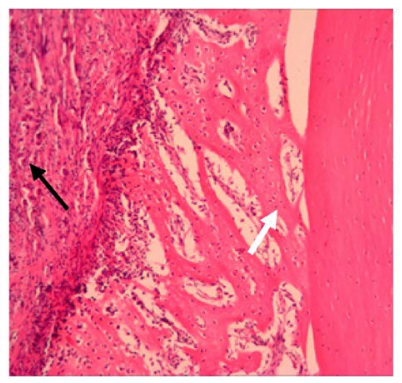

(c)

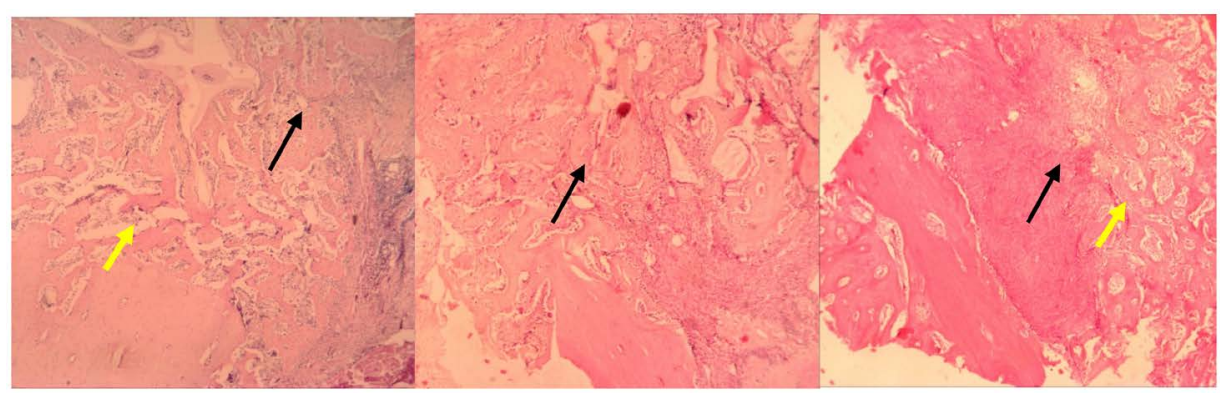

(d)

(e)

(f)

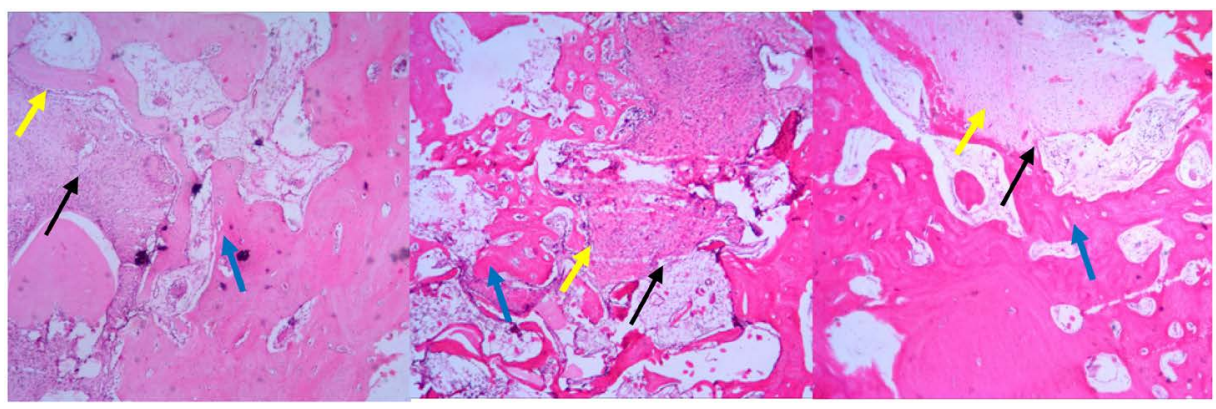

(g)

(h)

(i)

Figure 2. Microscopic view of scaffolds and grafts healing after 1, 2, and 12 weeks. Healing in BBM-hAMSC group showing mesenchymal and loose connective tissue at the periphery of scaffold (a); lack of mesenchymal and connective tissue noted in the periphery of scaffold in BBM group with the central zone dominated by PMN and mononuclear cells (b); mature trabecular and cortical bone with interstitial mesenchymal tissue and inflammatory cells in ABG group (c) (one week post implantation). Note: black arrow pointing to scaffold/graft peripheral zone, white arrow to central zone); Osteoid matrix with osteoblastic rimming seen in the central zone of scaffold in BBM-hAMSC group (d); islands of mesenchymal tissue blended with amorphous structure seen in the central zone of scaffold in BBM group (e); mature and immature bone were seen in ABG group (f) (2 weeks post implantation); Islands of trabeculae undergoing maturation contiguous with fibrous and mesenchymal tissue seen in the central zone of BBM-hAMSC (g); mesenchymal tissue contiguous with strands of bone trabeculae and amorphous structure were evident in BBM group (h); bone trabecula incorporating with resipient bone and interstitial mesenchymal tissue were seen in ABG group (i) (12 weeks post implantation). Note: black arrow pointing to mesenchymal tissue, yellow arrow to osteoid tissue, blue arrow to bony trabeculae.

\subsection{Examination of Early Phase of Bone Healing}

The mean of VEGF expression of BBM-hAMSC, BBM and ABG group in the first week were $0.76 \pm 0.43,0.92$ \pm 0.19 and $1.22 \pm 0.88$, respectively, which were found not to be statistically different. However, the mean of VEGF expression in the second week in BBM group $(2.04 \pm 0.35)$ were significantly higher compared to that in BBM-hAMSC and ABG group which were $0.50 \pm 0.51$ and $0.83 \pm 0.81$, respectively. The histogram pattern of VEGF expression within the first two weeks in BBM group was upward trend as opposed to downward in the BBM-hAMSC and ABG group (Figure 3).

The mean of angiogenesis in the first week in ABG group (7.18 \pm 0.83 ) was found to be significantly higher compared to that in BBM-hAMSC (4.06 \pm 4.25$)$ and BBM group (2.04 \pm 1.09$)$. However, the mean of angioge- 

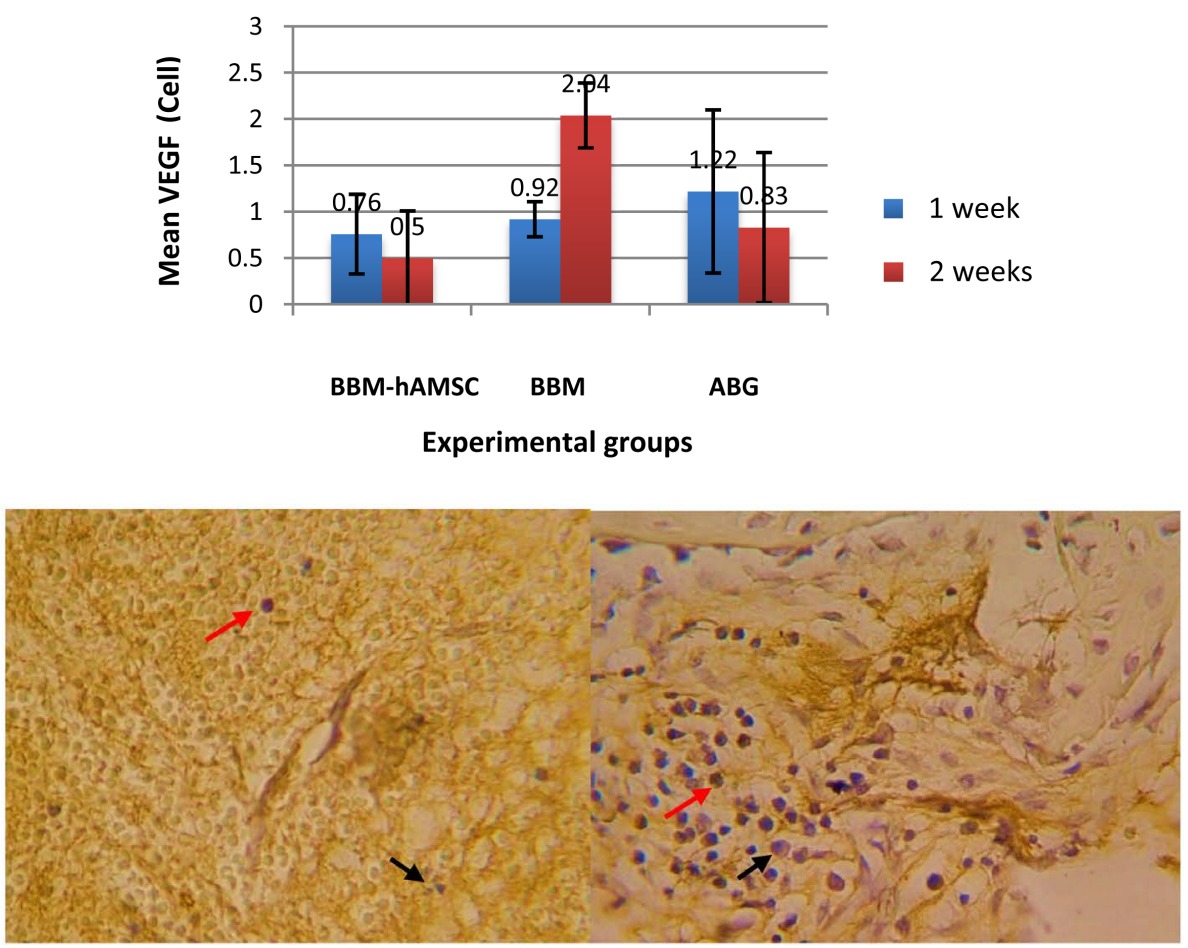

(a)

(b)

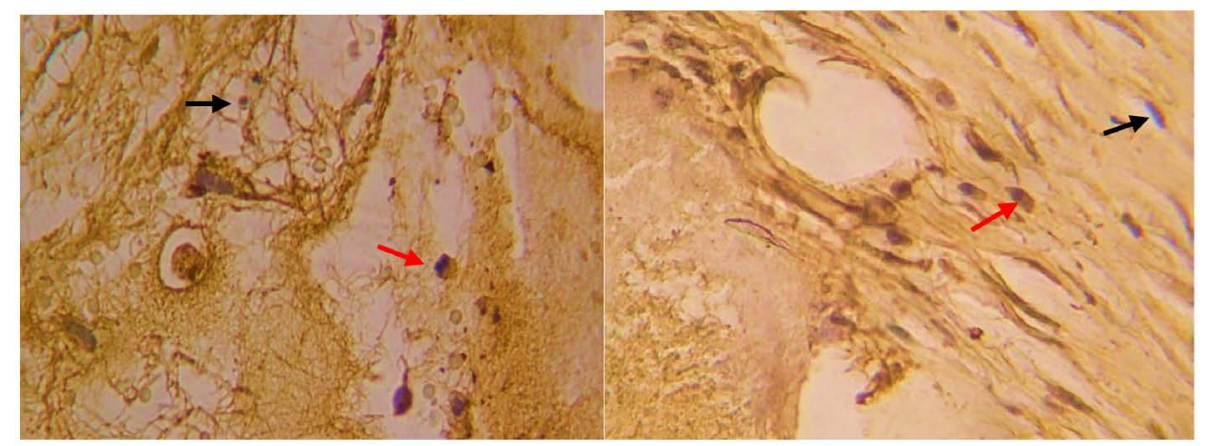

(c)

(d)

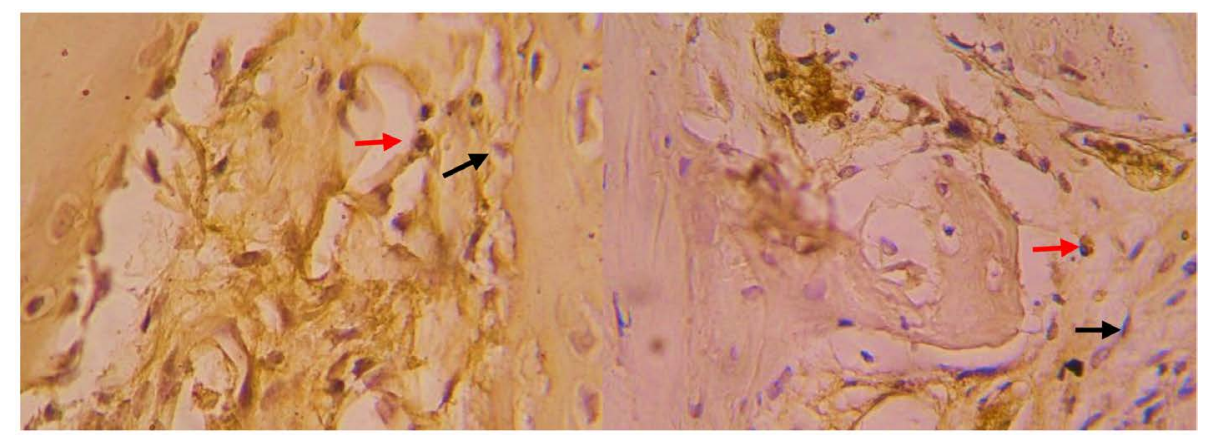

(e)

(f)

Figure 3. Result of immunohistochemistry for VEGF expression at early phase of bone healing. The mean of VEGF expression in all groups at one week were not statistically different, however, it was significantly higher in BBM group compared to BBM-hAMSC and ABG group; pattern of VEGF expression within the first two weeks in BBM group was upward trend as opposed to downward in the BBM-hAMSC and ABG group (top). (a) BBM-hAMSC group at 1 week; (b) BBM-hAMSC group at 2 weeks; (c) BBM group at 1 week; (d) BBM group at 2 week; (e) ABG group at 1 week; (f) ABG group at 2 week (note: red arrow pointing to positive cells, black arrow pointing to negative cells, $400 \times$ ). 
nesis in the second week were not significantly different among all of the groups. The histogram pattern of angiogenesis demonstrated upward trend in BBM group as opposed to downward trend in BBM-hAMSC and ABG group (Figure 4).

The mean of BMP2 expression in the first week in ABG group (1.06 \pm 0.13$)$ and BBM group $(0.74 \pm 0.22)$ were significantly higher than that in BBM-hAMSC $(0.48 \pm 0.20)$, however, in the second week the mean of BMP2 expression in BBM-hAMSC group $(1.00 \pm 0.18)$ and BBM group $(0.88 \pm 0.28)$ were significantly higher than that in ABG group $(0.51 \pm 0.25)$. The patterns of BMP2 expression in ABG group was downward compared to upward slope in tissue engineering groups (Figure 5).

The mean of Runx2 expression in the first week in ABG group (11.06 \pm 1.33$)$ was significantly higher than BBM-hAMSC and BBM group which were $1.78 \pm 0.70$ and $3.78 \pm 1.09$, respectively. The mean of Runx2 expression in the second week in BBM-hAMSC, BBM and ABG group were $9.63 \pm 3.67,6.10 \pm 0.39$ and $5.94 \pm$ 1.00 , respectively, which were found not to be statistically different. The histogram pattern of Runx2 expression in ABG group was downward compared to upward slope in BBM-hAMSC and BBM group (Figure 6).

\subsection{Examination of Late Phase of Bone Healing}

The result showed that the mean of Runx2 expression in twelfth week of healing in BBM-hAMSC, BBM and ABG group were $8.95 \pm 3.02,3.30 \pm 0.17$, and $6.83 \pm 1.00$, respectively (Figure 6). Statistical analysis showed that Runx2 expression in BBM-hAMSC and ABG group was not significantly different while both of them were significantly higher than that of BBM group. The histogram showed that Runx2 expression in twelfth week followed a downward trend in BBM-hAMSC and BBM group compared to stable pattern in ABG group.

The mean thickness of collagen type-I fibers in BBM-hAMSC, BBM and ABG group were $7.31 \pm 1.81 \mu \mathrm{m}$, $6.83 \pm 0.72 \mu \mathrm{m}$ and $3.75 \pm 0.46 \mu \mathrm{m}$, respectively (Figure 7). Statistical analysis indicated that the the collagen-I fibers thickness in BBM-hAMSC and BBM group were not significantly different, however, both significantly higher than that of ABG group. The mean of osteocalcin expression in BBM-hAMSC group (6.96 \pm 0.79 ) was significantly higher than that in BBM group (2.13 \pm 0.15$)$ and ABG group $(2.75 \pm 0.54)$ (Figure 7).

The result showed that the mean bony trabecula area in BBM-hAMSC $\left(204,090.25 \pm 103,187.16 \mu^{2}\right)$ and ABG group (149,472.50 $\pm 76,478.17 \mu^{2}$ ) were not significantly different, but they were relatively higher than that in BBM group $\left(86,256.67 \pm 62,903.83 \mu \mathrm{m}^{2}\right)$. The median of bone incorporation scoring between BBMhAMSC and ABG group were found to be not significantly different, but they were relatively higher than that in BBM group (Figure 8).

\section{Discussion}

This experimental study attempted to reveal the healing process and bone forming capacity of BBM scaffold loaded with hAMSC compared to autogenous bone graft in the reconstruction of a critical size mandibular bone defect. The healing mechanism was categorized into early phase, the tissue healing in the first and second weeks, and late phase which was the osteogenesis process at twelfth week observation. Few molecular events in the early tissue healing were evaluated. Expression of VEGF, the most potent angiogenic factor, amount of angiogenesis, expression of BMP2, the most important osteoinductive factor, and expression of Runx2, the specific marker of osteogenic differentiation, were evaluated in the early tissue healing phase. Few osteogenic parameters were evaluated in the late healing stage such as expression of Runx2, thickness of collagen type-I fibres, one of the indicators of matrix maturation, and osteocalcin, a specific marker of mature osteoblast, all of which were collectively referred to asosteogenic process. The evaluation of trabecular bone area and bone incorporation score were used to represent bone forming capacity or osteogenic capacity.

The histology findings at first week after implantation clearly showed somewhat prolonged inflammation and delayed tissue healing in the scaffold of BBM group. This could be strongly associated with the absence of MSC inside the scaffold of BBM as opposed to BBM-hAMSC group. It was likely that hAMSC loaded in the scaffold might have posed anti-inflammatory effects on the surrounding tissues. Result of few studies revealed that hAMSC possess an immunoprivileged status, which is in part due to the expression of low to moderate levels of surface MHC-I, and the presence of low levels or even absence of MHC II and costimulatory molecules [16] [17].

The VEGF expression in the first week of healing which was comparable among all groups but followed by downward trend in BBM-hAMSC and ABG group compared to upward trend in BBM group was interesting to 

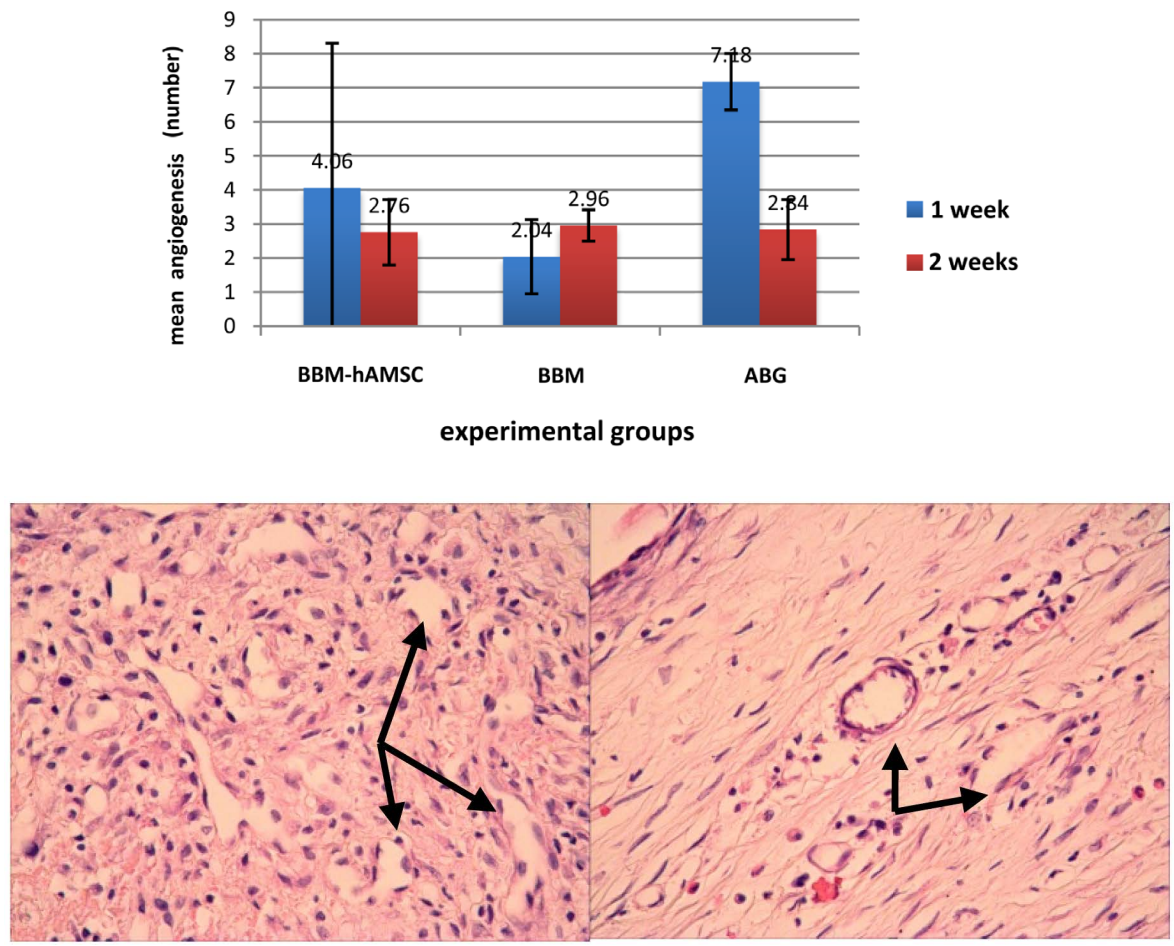

(a)

(b)

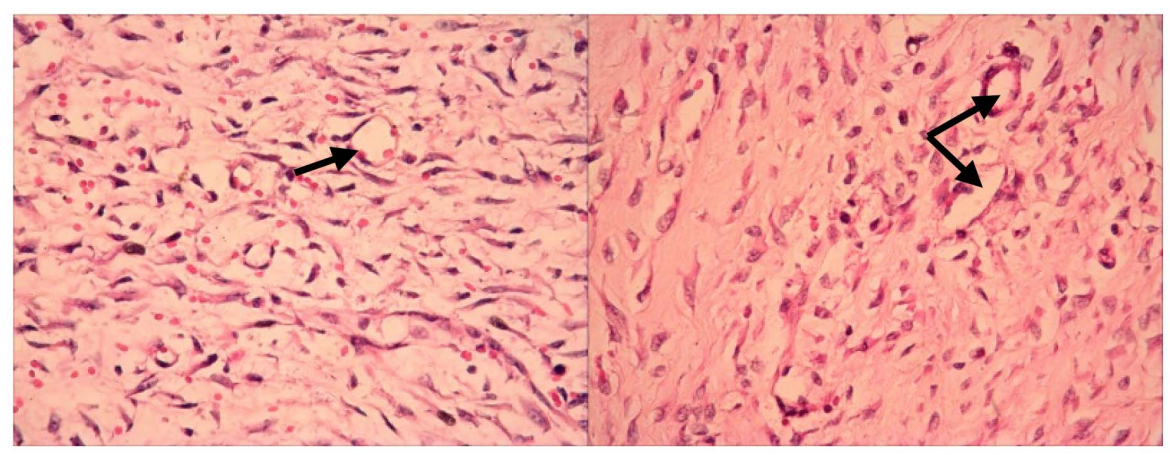

(c)

(d)

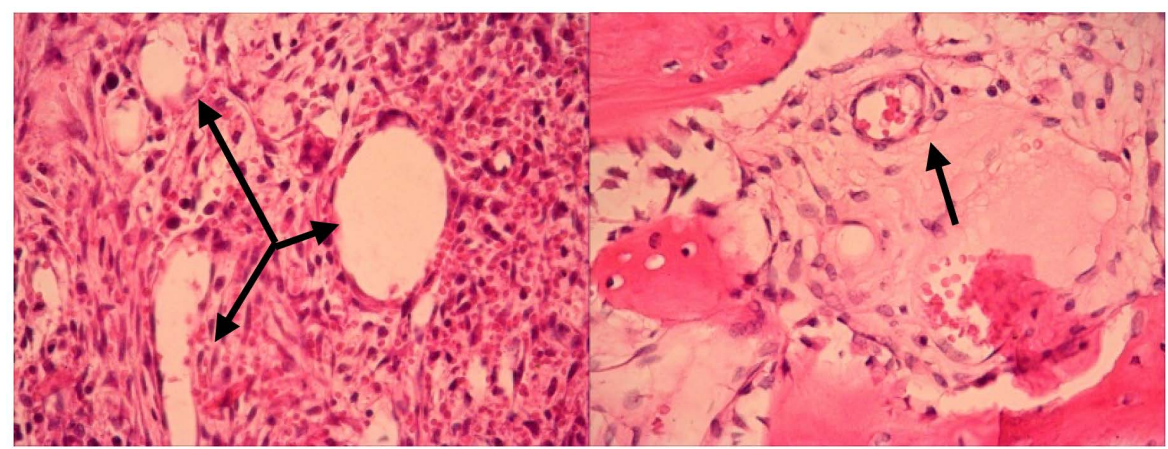

(e)

(f)

Figure 4. Result of histology evaluation for Angiogenesis. Mean of angiogenesis (number of arteriole, venoule, and capillary) at one week showed significantly higher number of vessels in ABG group compared to tissue engineering groups, while at two week they were somewhat comparable (top). Note: (a), (c), (e) are photos of angiogenesis in BBM-hAMSC, BBM and ABG group, respectively, at 1 week; (b), (d), (f) are photos of angiogenesis in BBM-hAMSC, BBM and ABG group, respectively, at 2 weeks (black arrow pointing to arteriole, HE staining, $\times 100)$. 

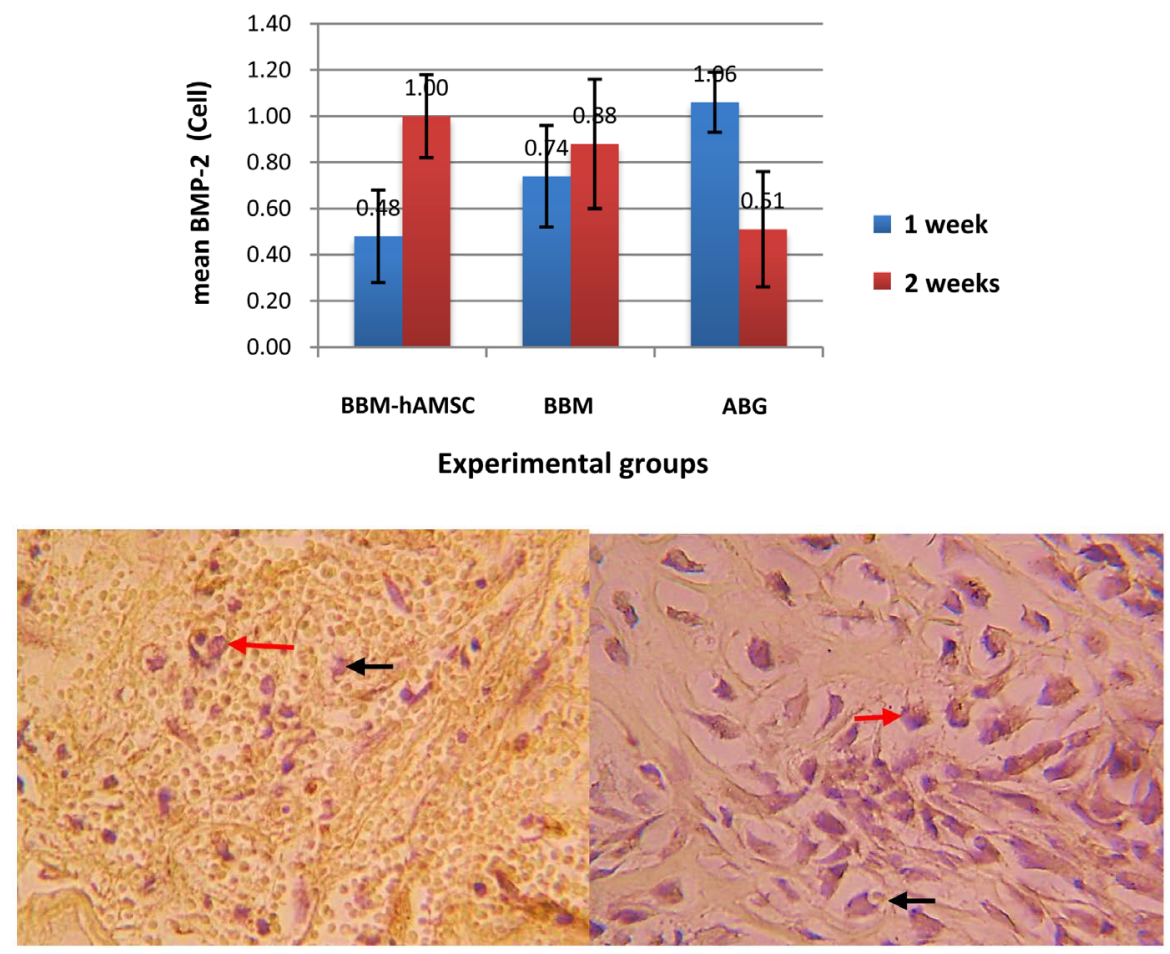

(a)

(b)

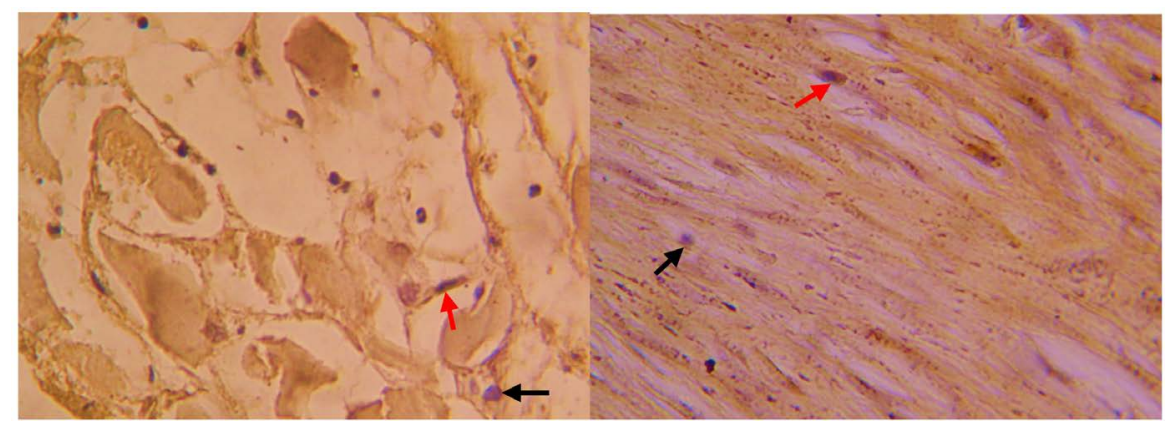

(c)

(d)

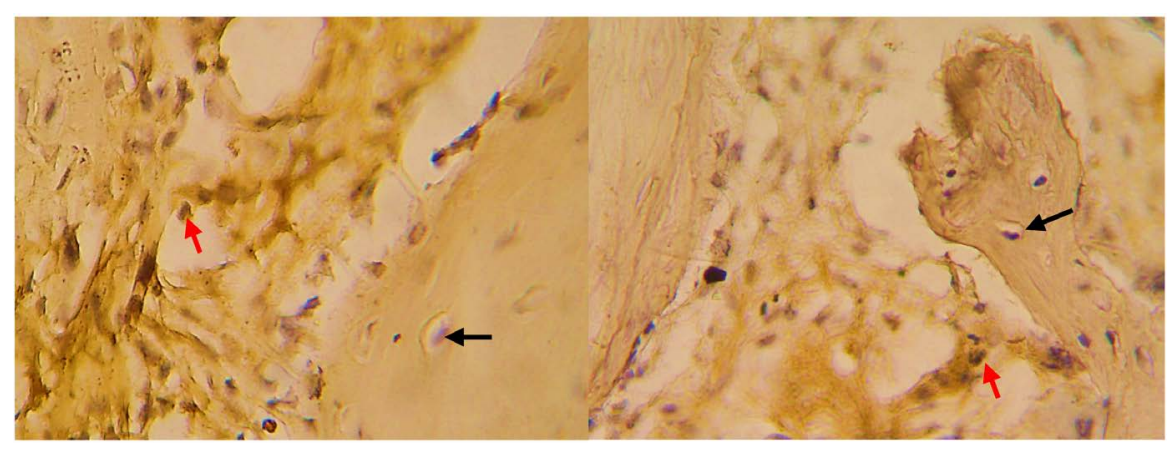

(e)

(f)

Figure 5. Result of immunohistochemistry evaluation for BMP2 expression at early phase of bone healing. The mean of BMP2 expression at one week in ABG and BBM group were significantly higher than BBM-hAMSC, while at two week they were significantly higher in BBM-hAMSC and BBM compared with ABG group. The pattern of BMP2 expression in ABG group was downward compared to upward slope in tissue engineering groups (top). (a) BBM-hAMSC group at 1 week; (b) BBM-hAMSC group at 2 weeks; (c) BBM group at 1 week; (d) BBM group at 2 week; (e) ABG group at 1 week (f) ABG group at 2 week (note: red arrow pointing to positive cells, black arrow pointing to negative cells, $\times 400$ ). 


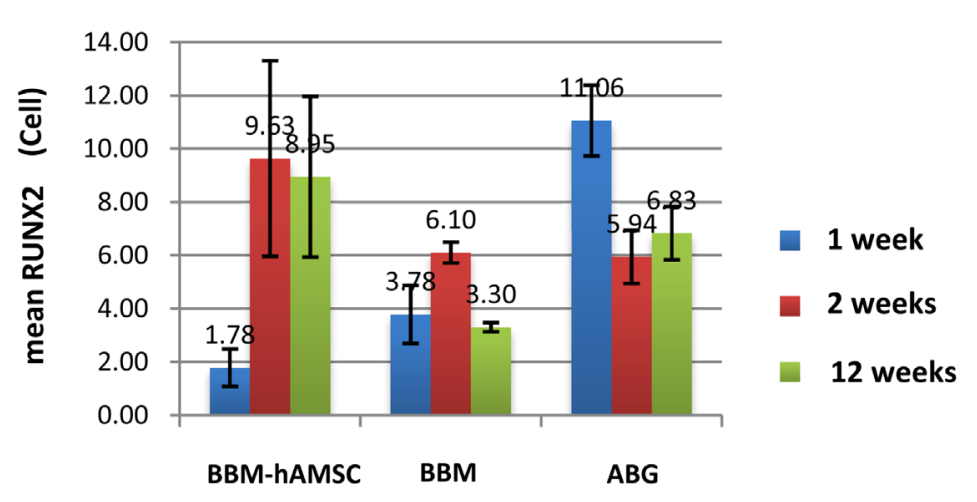

Experimental groups

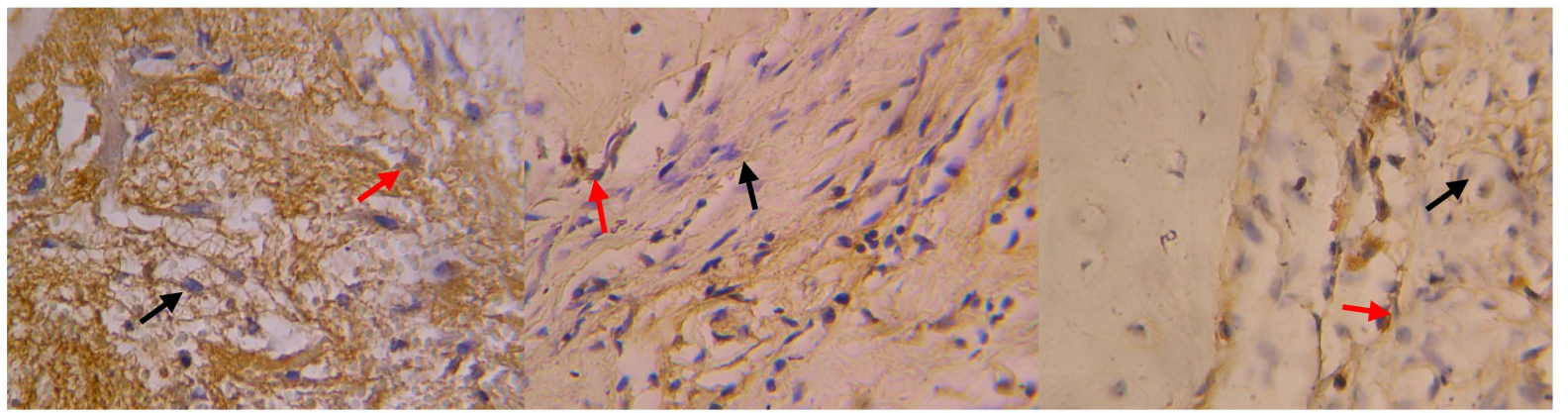

(a)

(b)

(c)

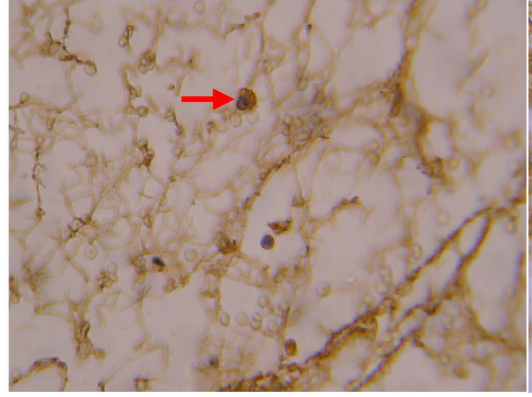

(d)

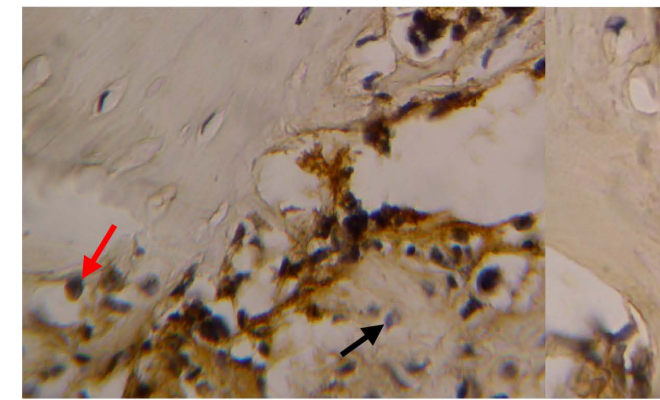

(g)

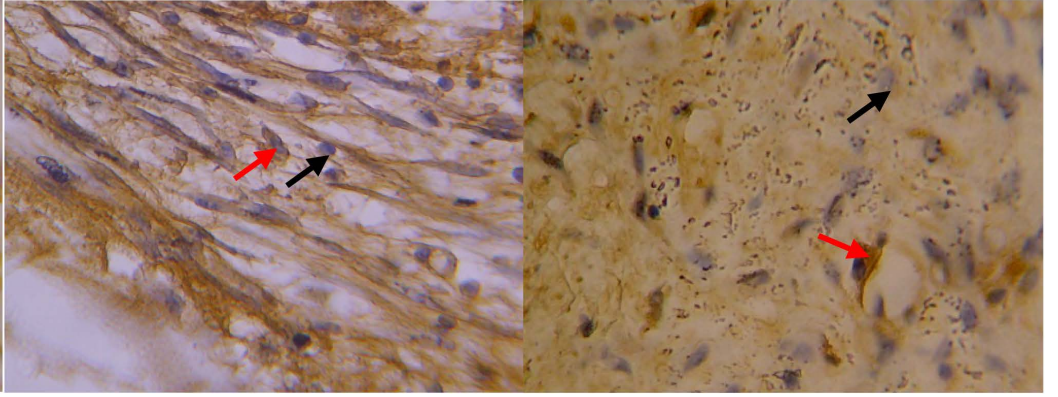

(e)

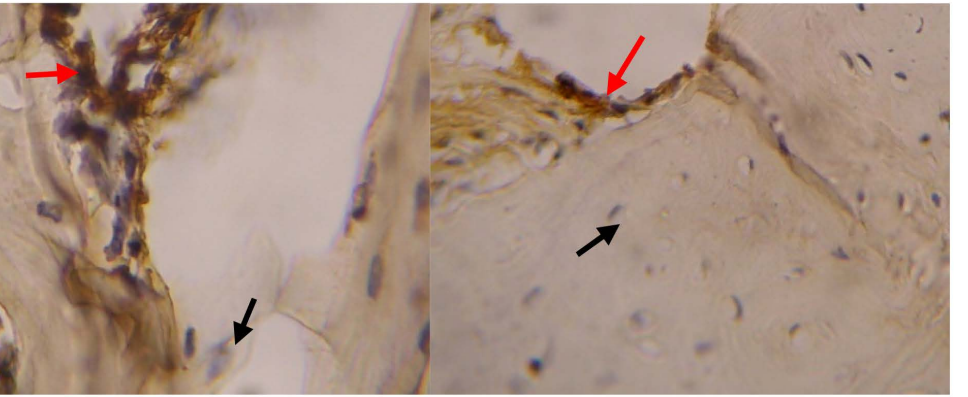

(f)

(i)

Figure 6. Result of immunohistochemistry evaluation forRunx2 expression at early and late phase of bone healing. The mean of Runx2 expression in the first week in ABG group was significantly higher than BBM-hAMSC and BBM group, in the second week they were not statistically different among all groups, while in the twelfth week they were as high in BBMhAMSC and ABG group which were significantly higher than BBM group (top). Photos (a), (b), (c) were BBM-hAMSC group at 1, 2, 12 week; (d), (e), (f) were BBM group at 1, 2, 12 week; (g), (h), (i) were ABG group at 1, 2, 12 week (note: red arrow pointing to positive cells, black arrow pointing to negative cells, $\times 400$ ). 

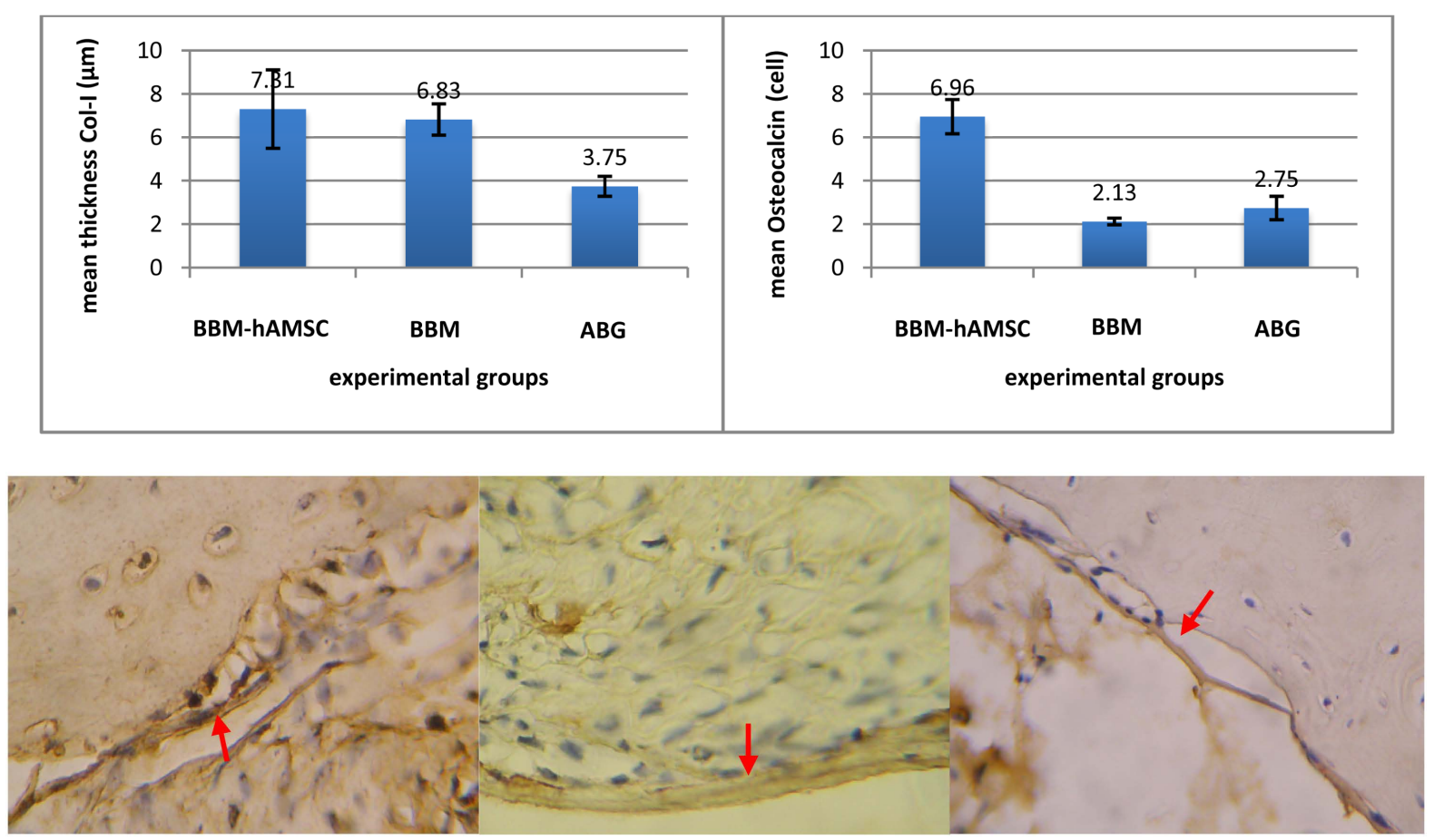

(a)

(b)

(c)

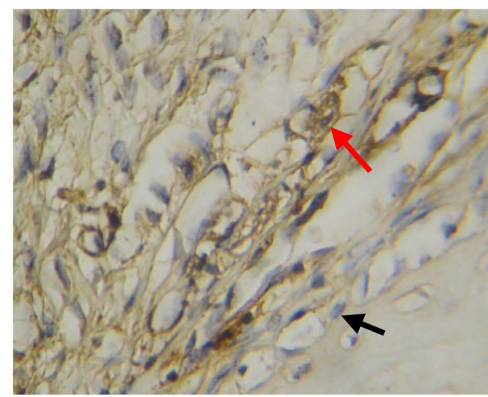

(d)

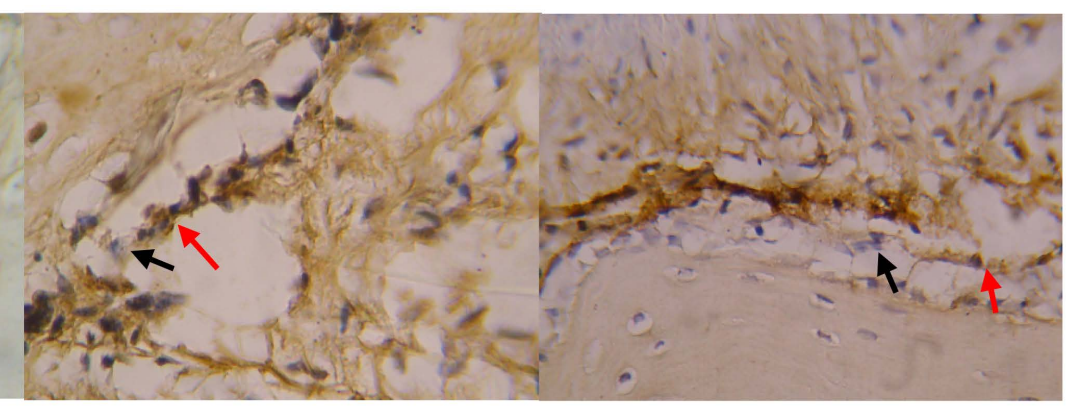

(e)

(f)

Figure 7. Result of immunohistochemistry evaluation for thickness of collagen type-I fibers and osteocalcin expression at 12 week. Thickness of collagen type-I fibers was significantly higher in tissue engineering groups compared to ABG group (top left), expression of osteocalcin was significantly higher in BBM-hAMSC group compared to BBM and ABG group (top right). Photos (a), (b) and (c) showing collagen type-I fibers in BBM-hAMSC, BBM and ABG group, respectively (red arrow pointing to collagen type-I fibers, $\times 400$ ); photos (d), (e) and (f) showing osteocalcin expression in BBM-hAMSC, $\mathrm{BBM}$ and $\mathrm{ABG}$ group, respectively(red arrow pointing to positive cells, black arrow pointing to negative cells, $\times 400$ ).

note. This finding indicated that VEGF expression in BBM-hAMSC and ABG group might have reached their peaks in the first few days after implantation compared to that in BBM group which started at later stage. This phenomenon was likely to be associated with the presence of living cells inside the scaffold of BBM-hAMSC group and the grafted bone in ABG group. As the BBM scaffold and the non-vascularized bone graft were avascular and hence hypoxic, overexpression of VEGF by the pre-existing cells in those groups was expected to occur in the first few post-implantation days. This presumption was supported by result of the study which demonstrated that exposure of stem cells to hypoxia resulted in up-regulation of VEGF [18]. Further study evaluating VEGF expression in implanted scaffold earlier than 7 days would be required to prove this presumption.

The expression of BMP2 and Runx2 in ABG group which were significantly higher than the tissue engineering groups in the first week after implantation and the decline of those parameters in ABG group as opposed to the increase of the same parameters in tissue engineering groups in the second week (Figure 4 and Figure 5) suggested that osteogenic activities was initiated earlier and stronger in ABG compared to tissue engineering 

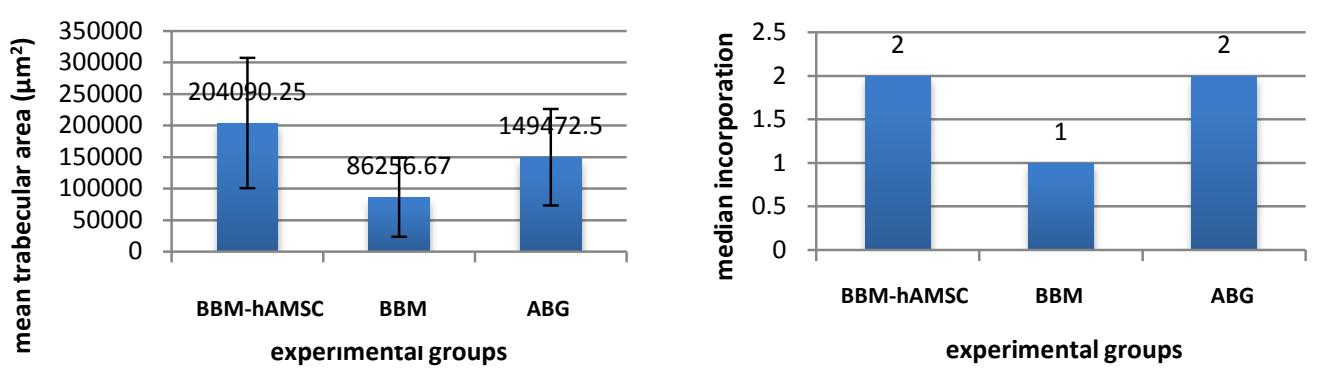

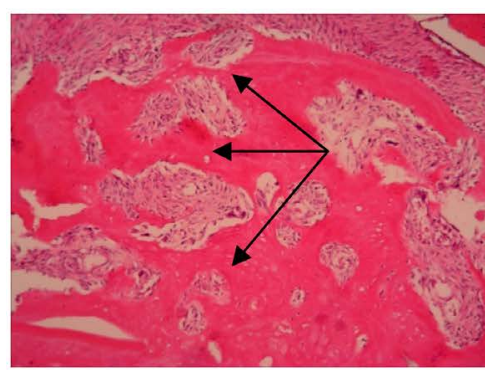

(a)

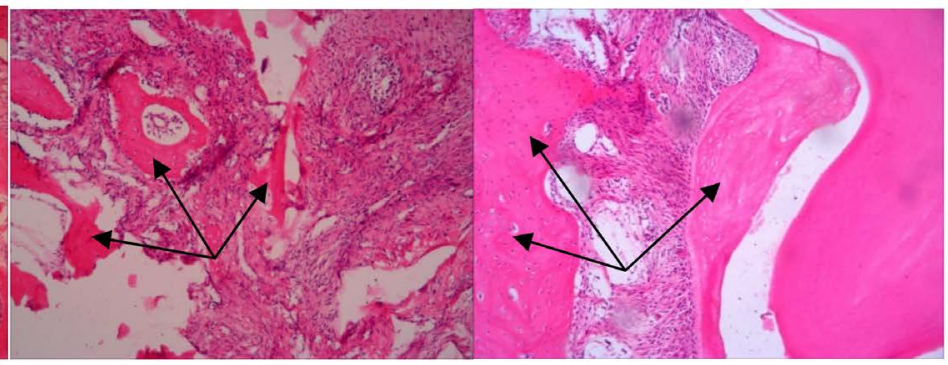

(b)

(c)

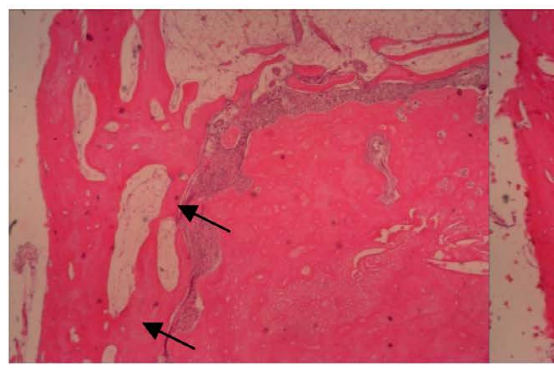

(d)

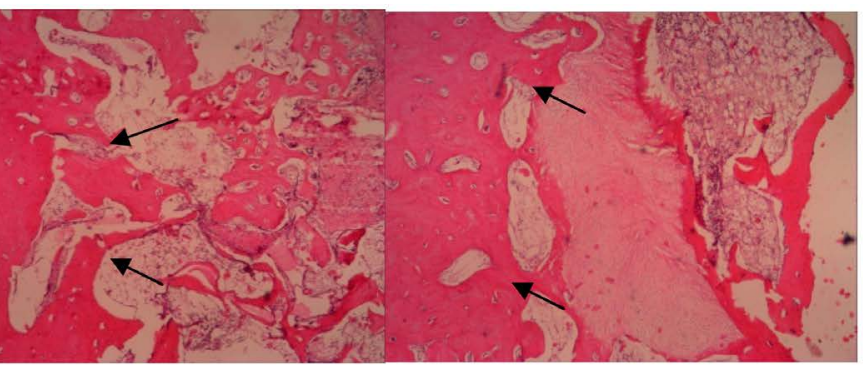

(e)

(f)

Figure 8. Result of histology evaluation for trabecular area and bone incorporation at 12 weeks. The mean trabecular area in BBM-hAMSC and ABG group were significantly higher than BBM group (top left); the median of bone incorporation score in BBM-hAMSC and ABG group were relatively higher than BBM group (top right). Note. Photos (a), (b), and (c) showing trabecular area in BBM-hAMSC, BBM and ABG group, respectively (black arrow pointing to bone trabeculae, HE staining, $\times 40$ ); Photos (d), (e) and (f) showing bone incorporation in BBM-hAMSC, BBM and ABG group, respectively (black arrow pointing to incorporation area between new bone and resipient bone, HE staining, $\times 40)$.

groups. The similar pattern of BMP2 and Runx2 expressions in all experimental groups (Figure 4 and Figure 5) suggested that their expressions might be strongly correlated. This findings also confirmed that BMP2 was the most important osteoinductive factor involved in the process of bone healing as suggested by some authors [19] [20].

It was interesting to note that expression of BMP2 in BBM-hAMSC group in the first week of healing was lower compared to that in BBM group which actually was unexpected as the presence of hAMSC in the former group should have resulted in higher BMP2 expression. This phenomenon could be associated with the high expression of VEGF by hAMSC in the first week of healing inhibiting BMP2 expression. This presumption was supported by the result of in vitro studies which demonstrated that VEGF was a potent inhibitor of BMP, and overexpression of VEGF suppressed the in vitro differentiation and osteogenesis of MSC [21] [22]. The overall results discussed above indicated that angiogenesis and osteogenic differentiation started earlier in ABG group in the first week of healing but became comparable with BBM-hAMSC in the following week.

Expression of Runx2, thickness of collagen type-I fibres, and expression of osteocalcin in the twelfth week represented the amount of mesenchymal tissue differentiating towards osteoblastic lineage, osteoid matrix formation, and matrix undergoing mineralization forming bone trabecula, respectively. The more mature the osteogenic tissue in one specific compartment the more tissue shifting to the right and vice versa. The result of evaluation of Runx2 expression, thickness of collagen type-I fibres, and expression of osteocalcin indicated that 
osteogenic process were still ensuing in all of the three groups at the end of twelfth week. The result also indicated that although the level of osteogenic maturation was histologically higher in ABG group compared to BBM-hAMSC, the magnitude of osteogenic process in ABG group was significantly lower than BBM-hAMSC group. It was likely because the magnitude of osteogenic process in ABG group was dependent on that of resorption process of the graft by osteoclast activity which was not evaluated in this study. This assumption was supported by "remodelling cycle" theory [23] which suggested that resorption of autogenous bone graft might last until several months after implantation. The result of evaluation of trabecular area and bone incorporation at twelfth weeks after implantation indicated that the bone forming capacity or osteogenic capacity of BBMhAMSC group was comparable to that of ABG group.

\section{Conclusion}

This study concluded that early healing activities of critical size mandibular defect were higher in autogenous bone graft than in BBM-hAMSC, while osteogenic activities in the late stage of healing were, on the other hand, higher in BBM-hAMSC compared to autogenous bone graft. It was also concluded that bone forming capacity, or osteogenic capacity, of BBM-hAMSC was comparable to autogenous bone graft in the reconstruction of critical size defect in the mandible.

\section{Acknowledgements}

This work was supported by grants from DIPA BOPTN, Ministry of Education, Republic of Indonesia, Fiscal Year 2014. This study was made possible by collaboration of Faculty of Dental Medicine Airlangga University, Laboratory of Stem Cell Institute of Tropical Disease Airlangga University, and Installation of Biomaterial Tissue Bank and Stem Cell Dr. Soetomo General Hospital Surabaya.

\section{References}

[1] Arrington, E.D., Smith, W.J., Chambers, H.G., Bucknell, A.L. and Davino, N.A. (1996) Complications of Iliac Crest Bone Graft Harvesting. Clinical Orthopaedics and Related Research, 329, 300-309. http://dx.doi.org/10.1097/00003086-199608000-00037

[2] Heary, R.F., Schlenk, R.P., Sacchieri, T.A., Barone, D. and Brotea, C. (2002) Persistent Iliac Crest Donor Site Pain: Independent Outcome Assessment. Neurosurgery, 50, 510-516.

[3] Bruder, S.P., Kraus, K.H., Goldberg, V.M. and Kadiyala, S. (1998) The Effect of Implants Loaded with Autologous Mesenchymal Stem Cells on the Healing of Canine Segmental Bone Defects. Journal of Bone \& Joint Surgery (American Volume), 80, 985-996.

[4] Kon, E., Muraglia, A., Corsi, A., Bianco, P., Marcacci, M. and Martin, I. (2000) Autologous Bone Marrow Stromal Cells Loaded onto Porous Hydroxyapatite Ceramic Accelerate Bone Repair in Critical-Size Defects of Sheep Long Bones. Journal of Biomedical Materials Research, 49, 328-337. http://dx.doi.org/10.1002/(SICI)1097-4636(20000305)49:3<328::AID-JBM5>3.0.CO;2-Q

[5] Petite, H., Viateau, V., Bensaid, W., Meunier, A., de Pollak, C. and Bourguignon, M. (2000) Tissue-Engineered Bone Regeneration. Nature Biotechnology, 9, 959-963. http://dx.doi.org/10.1038/79449

[6] Mastrogiacomo, M., Corsi, A., Francioso, E., Di Comite, M., Monetti, F., Scaglione, S., Favia, A., Crovace, A., Bianco, P. and Cancedda, R. (2006) Reconstruction of Extensive Long Bone Defects in Sheep Using Resorbable Bioceramics Based on Silicon Stabilized Tricalciumphosphate. Tissue Engineering, 12, 1261-1273. http://dx.doi.org/10.1089/ten.2006.12.1261

[7] Kruyt, M.C., Wilson, C.E., de Bruijn, J.D., van Blitterswijk, C.A., Oner, C.F. and Verbout, A.J. (2006) The Effect of Cell-Based Bone Tissue Engineering in a Goat Transverse Process Model. Biomaterials, 29, 5099-5106. http://dx.doi.org/10.1016/j.biomaterials.2006.05.048

[8] Yue, H., Zhang, Z.Y., Zhu, H.G., Qiu, W.L., Jiang, X.Q. and Guo, W. (2007) Experimental Study on Reconstruction of Segmental Mandible Defects Using Tissue Engineered Bone Combined Bone Marrow Stromal Cells with ThreeDimensional Tricalcium Phosphate. Journal of Craniofacial Surgery, 18, 800-805. http://dx.doi.org/10.1097/scs.0b013e31806901f5

[9] Yuan, J., Cui, L., Zhang, W.J., Liu, W. and Cao, Y.L. (2007) Repair of Canine Mandibular Bone Defects with Bone Marrow Stromal Cells and Porous Beta Tricalcium Phosphate. Biomaterials, 28, 1005-1013. http://dx.doi.org/10.1016/j.biomaterials.2006.10.015

[10] Sakaguchi, Y., Sekiya, I. and Yagishita, K. (2004) Suspended Cells from Trabecular Bone by Collagenase Digestion 
Become Virtually Identical to Mesenchymal Stem Cells Obtained from Marrow Aspirates. Blood, 104, 2728-2735. http://dx.doi.org/10.1182/blood-2003-12-4452

[11] Miki, T., Lehmann, T., Cai, H., Stolz, D.B. and Strom, S.C. (2005) Stem Cell Characteristics of Amniotic Epithelial Cells. Stem Cells, 23, 1549-1559. http://dx.doi.org/10.1634/stemcells.2004-0357

[12] Kim, J., Kang, H.M., Kim, H., Kim, M.R., Kwon, H.C. and Gye, M.C. (2007) Ex Vivo Characteristics of Human Amniotic Membrane-Derived Stem Cells. Cloning and Stem Cells, 9, 581-594. http://dx.doi.org/10.1089/clo.2007.0027

[13] Portmann-Lanz, C.B., Schoeberlein, A., Huber, A., Sager, R., Malek, A. and Holzgreve, W. (2006) Placental Mesenchymal Stem Cells as Potential Autologous Graft for Pre- and Perinatal Neuroregeneration. American Journal of Obstetrics and Gynecology, 194, 664-673. http://dx.doi.org/10.1016/j.ajog.2006.01.101

[14] Ilancheran, S., Michalska, A., Peh, G., Wallace, E.M., Pera, M. and Manuelpillai, U. (2007) Stem Cells Derived from Human Fetal Membranes Display Multilineage Differentiation Potential. Biology of Reproduction, 77, 577-588. http://dx.doi.org/10.1095/biolreprod.106.055244

[15] Kamadjaja, D.B., Purwati, Ferdiansyah, Rantam, F.A. and Pramono, C. (2014) The Osteogenic Capacity of Human Amniotic Membrane Mesenchyma Stem Cell (hAMSC) and Potential for Applicationin Maxillofacial Bone Reconstruction in Vitro Study. Journal of Biomedical Science and Engineering, 7, 497-503. http://dx.doi.org/10.4236/jbise.2014.78051

[16] Lindenmair, A., Hatlapatka, T., Kollwig, G., Hennerbichler, S., Gabriel, C., Wolbank, S., Redl, H. and Kasper, C. (2012) Mesenchymal Stem or Stromal Cells from Amnion and Umbilical Cord Tissue and Their Potential for Clinical Applications. Cells, 1, 1061-1088. http://dx.doi.org/10.3390/cells1041061

[17] Shi, Y., Su, J., Roberts, A.I., Shou, P., Rabson, A.B. and Ren, G. (2012) How Mesenchymal Stem Cells Interact with Tissue Immune Responses. Trends in Immunology, 33, 136-143. http://dx.doi.org/10.1016/j.it.2011.11.004

[18] Potier, E., Ferreira, E., Andriamanalijaona, R., Pujol, J.P., Oudina, K., Logeart-Avramoglou, D. and Petite, H. (2007) Hypoxia Affects Mesenchymal Stromal Cell Osteogenic Differentiation and Angiogenic Factor Expression. Bone, 40, 1078-1087. http://dx.doi.org/10.1016/j.bone.2006.11.024

[19] Hanada, K., Solchaga, L.A., Caplan, A.I., Hering, T.M., Goldberg, V.M., Yoo, J.U. and Johnstone, B. (2001) BMP-2 Induction and TGF-Beta-1 Modulation of Rat Periosteal Cell Chondrogenesis. Journal of Cellular Biochemistry, 81, 284-294. http://dx.doi.org/10.1002/1097-4644(20010501)81:2<284::AID-JCB1043>3.0.CO;2-D

[20] Wang, F.S., Yang, K.D., Kuo, Y.R., Wang, C.J., Sheen-Chen, S.M., Huang, H.C. and Chen, Y.J. (2003) Temporral and Spatial Expression of Bone Morphogenetic Proteins in Extracorporeal Shock Wave-Promoted Healing of Segmental Defect. Bone, 32, 387-396. http://dx.doi.org/10.1016/S8756-3282(03)00029-2

[21] Schonmeyr, B.H., Soares, M., Avraham, T., Clavin, N.W., Gewalli, F. and Mehrara, B.J. (2010) Vascular Endothelial Growth Factor Inhibit Bone Morphogenetic Protein 2 Expression in Rat Mesenchymal Stem Cells. Tissue Engineering Part A, 16, 653-662. http://dx.doi.org/10.1089/ten.tea.2009.0426

[22] Lin, Z.W., Wang, J.S., Lin, L.J., Zhang, J.W., Liu, Y.L., Ming, S. and Qi, L. (2014) Effects of BMP2 and VEGF165 on the Osteogenic Differentiation of Rat Bone Marrow-Derived Mesenchymal Stem Cells. Experimental and Therapeutic Medicine, 7, 625-629.

[23] Friedlaender, G. (1987) Current Concept Review, Bone Grafts: The Basic Science Rationale for Clinical Applications. The Journal of Bone \& Joint Surgery, 69, 786-790. 